\title{
Effect of cobalt particle deposition on quantum corrections to Drude conductivity in twisted CVD graphene
}

\author{
Alexander K. Fedotov ${ }^{1}$, Sergey L. Prishchepa ${ }^{2,3}$, Alexander S. Fedotov ${ }^{4}$, \\ Vladzislaw E. Gumennik ${ }^{1,4}$, Ivan V. Komissarov ${ }^{2,3}$, Artem O. Konakov ${ }^{5}$, \\ Svetlana A. Vorobyova ${ }^{5}$, Oleg A. Ivashkevich ${ }^{5}$, Andrei A. Kharchenko ${ }^{1}$ \\ 1 Research Scientific Institute for Nuclear Problems of Belarusian State University, 11 Bobruiskaya Str., Minsk, 220030, Belarus \\ 2 Belarusian State University of Informatics and Radioelectronics, 6 P. Brovka Str., Minsk, 220013, Belarus \\ 3 National Research Nuclear University MEPhI, 31 Kashirskoe Shosse, Moscow, 115409, Russia \\ 4 Belarusian State University, 4 Nezavisimosti Ave., Minsk, 220030, Belarus \\ 5 Research Institute for Physical Chemical Problems of the Belarusian State University, 14 Leningradskaya Str., Minsk, 220006, Belarus \\ Corresponding author: Andrei A. Kharchenko (XaaTM@mail.ru)
}

Received 3 December 2019 • Accepted 29 December 2019 • Published 31 December 2019

Citation: Fedotov AK, Prishchepa SL, Fedotov AS, Gumennik VE, Komissarov IV, Konakov AO, Vorobyova SA, Ivashkevich OA, Kharchenko AA (2019) Effect of cobalt particle deposition on quantum corrections to Drude conductivity in twisted CVD graphene. Modern Electronic Materials 5(4): 165-173. https://doi.org/10.3897/j.moem.5.4.52068

\begin{abstract}
Graphene applications in electronics require experimental study of the formation of high-quality Ohmic contacts and deeper understanding of electron transport mechanisms at metal/grapheme contacts. We have studied carrier transport in twisted CVD graphene decorated with electrodeposited Co particles forming Ohmic contacts with graphene layers. We have compared layer resistivity as a function of temperature and magnetic field $R_{\square}(T, B)$ for as-synthesized and decorated twisted graphene on silicon oxide substrates. Experiments have proven the existence of negative (induction $<1 \mathrm{Tl}$ ) and positive (induction $>1 \mathrm{Tl}$ ) contributions to magnetoresistance in both specimen types. The $R_{\square}(T, B)$ functions have been analyzed based on the theory of $2 \mathrm{D}$ quantum interference corrections to Drude conductivity taking into account competition of hopping conductivity mechanism. We show that for the experimental temperature range $(2-300 \mathrm{~K})$ and magnetic field range (up to $8 \mathrm{Tl}$ ), carrier transport description in test graphene requires taking into account at least three interference contributions to conductivity, i.e., from weak localization, intervalley scattering and pseudospin chirality, as well as graphene buckling induced by thermal fluctuations.
\end{abstract}

\section{Keywords}

graphene, graphene/metal structures, electron transport, magnetostriction

\section{Introduction}

Graphenes have been intensely investigated in the last decade due to their specific physical properties such as high electrical and heat conductivities, well-developed surface, high mechanical strength, elasticity etc. In accordance with the graphene electronics development roadmap [1] the combination of these properties shows good promise 
for the development of hybrid nanostructures (e.g., metal/ graphene) for new types of sensors, transducers, memristors, spintronic devices, energy storage devices, tools for magnetic visualization of biological objects etc. [1-3]. One of the most promising approaches to the synthesis of these composite structures is the deposition of particles of different magnetic and nonmagnetic metals on graphene layer surfaces [4-6]. However one of the key problems to graphene use in electronics is the complexity of fabricating Ohmic contacts without damaging graphene crystal lattice. Experiments have shown that Ohmic contacts can be most easily formed on multilayered graphene [2, 3, $7,8]$. Nevertheless the problem of fabricating high-quality electric contacts remains a problem of attention for fundamental and technical research thus intensifying the importance of understanding carrier transport mechanisms near and through metal/graphene contacts.

Carrier transport in metal/graphene nanostructures depends on multiple factors. These are primarily the synthesis method (mechanical debonding, CVD, epitaxy etc.) and graphene type (single-layered, multilayered, twisted). Secondly one should take into account the type of dielectric substrate (quartz, glass, silicon oxide ctc.) onto which graphene is deposited or transferred. Finally important factors are the type, concentration and distribution of potential defects in graphene layers including polycrystalline ones as well as the properties of graphene / electric contact interfaces. These properties are mainly determined by deposited metal type and deposition technology $[1-3,9]$. Despite the large number of CVD graphene structure studies there are but scarce data on the electronic and galvanomagnetic properties of specimens in the form of metallic islands on graphene. There are studies of the effect of electrodeposited cobalt nanoparticles on the properties of twisted graphene [6, 9, 10, 11, 13, 14]. Photoelectron spectroscopy and magnetometry showed that electrodeposited cobalt particles undergo intense surface oxidation and preferentially form Co core / $\mathrm{CoO}$ shell structures. Nevertheless electrodeposition is helpful in the fabrication of Ohmic contacts [14].

In spite of extensive research into the galvanomagnetic properties of graphene layers and graphene/metal hybrid structures, there is still one more problem concerning understanding the main electrotransport mechanisms in graphene. Most of this research has dealt with micron sized graphene specimens produced either by debonding from graphite [12, 15-18] or cutting from single polycrystalline graphene grains [19-21]. However carrier transport in magnetic fields for large specimens (millimeters or fractions of a millimeter) has been dealt with in only a few works [22-26]. Obviously there is a great contribution to carrier transport in these specimens from grain boundaries and other large defects (ruptures, buckling etc.).

Descriptions of low-temperature electrotransport and magnetotransport in pure graphene available in literature most often adhere to interference mechanisms within the theory of quantum corrections to Drude conductivity under weak localization conditions [16-18, 27]. For strong localization one has to give preference to a hopping conductivity mechanism obeying the Mott [28] or Shklovsky-Efros [29] models for a zero magnetic field or the Mikoshiba [30] or Altshuler-Aronov-Khmelnitsky [27] models for electrotransport in an external magnetic field. Medium localization (between weak and strong) requires both interference and hopping transport mechanisms be taken into account [19-21]. Metallic particles (islands) deposited onto composite structures containing graphene layers may form spatially distributed (defragmented) metallic bridges $[14,31]$ which can bend current paths in graphene layers in a transverse magnetic field (through the effect of Lorentz force) and trigger the contribution of extraordinary magnetoresistance effect to magnetotransport [32]. Obviously the combination of the abovementioned transport mechanisms in metal/ graphene structures will be determined by defect density, graphene synthesis method and metal/graphene layer interface quality.

The aim of this work was to study the effect of cobalt particles on magnetotransport mechanisms in metal/graphene composite structures synthesized by electrodeposition of cobalt particles on twisted CVD graphene.

\section{Experimental}

We chose twisted graphene as the working layer for Co particle deposition since it is less affected by substrate as compared to single-layer graphene [31, 33]. Furthermore as noted above the possibility of producing an Ohmic contact between a metal and two-layered graphene is usually higher than for single-layer graphene $[2,3,7,8]$.

The growth conditions of the experimental graphene layers were described in detail earlier [14, 31, 33, 34]. The synthesis method was low atmospheric pressure chemical vapor deposition onto a $25 \mu \mathrm{m}$ thick copper foil (Alfa Aesar $99.8 \%$ purity) from $\mathrm{C}_{10} \mathrm{H}_{22} n$-decane as precursor with nitrogen as carrier gas. Co particles were electrodeposited onto the graphene layer surface on the copper foil at a $2.5 \mathrm{~mA} / \mathrm{cm}^{2}$ direct current for $30 \mathrm{~s}$ at room temperature. The electrolyte contained $0.96 \mathrm{~g} / \mathrm{l}$ cobalt sulfate and $0.064 \mathrm{~g} / 1$ sodium chloride solution in distilled water. The graphene specimens on the copper foil with electrodeposited Co particles were carefully rinsed in distilled water and dried at room temperature. For measuring the electrical properties of the graphene layers before and after Co deposition the graphene layers were transferred from the copper foil to oxidized silicon substrates after dissolving the copper foil in $1 \mathrm{M} \mathrm{FeCl}_{3}$ and rinsing graphene layer in distilled water.

The surface structure of the specimens on the copper foil and on the $\mathrm{SiO}_{2} / \mathrm{Si}$ substrates was examined under a Hitachi S4800 scanning electron microscope (SEM) equipped with a Bruker QUANTAX 200 energy dispersive X-ray spectrometer (EDXS) allowing elemental composition mapping. The quality of the graphene layer was also studied by Raman spectroscopy on a Confotec 
NR500 selected are Raman instrument with a $473 \mathrm{~nm}$ excitation wavelength and an approx. $3 \mathrm{~cm}^{-1}$ spectral resolution [14, 33-36]. Additionally the thickness of graphene transferred to glass was measured by $400-800 \mathrm{~nm}$ light transmittance (PROSCAN MC-121 spectrometer). The transmission ratio of graphene was $>94 \%$ at $550 \mathrm{~nm}$, thus the average number of graphene layers was two.

The electrical resistivity of graphene as a function of temperature and magnetic field $R(T, B)$ was measured with the four-probe method on a Cryogenics Ltd. cryogen-free measurement system (CFMS) with a closed cycle cryostat. The $R_{-}(T, B)$ functions were measured in the $2<T<300 \mathrm{~K}$ range in a transverse magnetic field of up to $8 \mathrm{Tl}$. The test specimens were placed on a contact pad as shown in Fig. $1 a$ with 4 indium (In) electric contacts into which $50 \mu \mathrm{m}$ diam. copper microwires were soldered. The measurement cell with the specimen on the contact pad inside was placed into a measurement probe in a low pressure helium gas atmosphere. The probe incorporated thermometers, magnetic field gages, heaters and heat screens. The probe was inserted into the superconducting solenoid channel inside the CFMS cryostat. The current applied to the specimen was generated and measured by a Keithley 6430 meter allowing electrical resistivity measurements within the $100 \mu \mathrm{Ohm}$ to $10 \mathrm{GOhm}$ range accurate to within $0.1 \%$. The specimen temperature was controlled with LakeShore diodes calibrated accurate to $0.005 \mathrm{~K}$ with a reproducibility of not worse than $0.01 \mathrm{~K}$. The specimen temperature was stabilized and measured with a LakeShore 331 controller.

The layer conductivity of graphene was calculated as follows:

$$
\sigma_{\square}(T)=\sigma(T)\left(\frac{L}{W}\right),
$$

where $\sigma_{\square}(T)$ is the specimen conductivity, $W$ is the conducting channel (graphene layer) width and $L$ is the distance between the voltage contacts 3 and 4 in Fig. $1 b$. The relative magnetoresistance was calculated as follows:

$$
M R=100 \frac{R(B)-R(0)}{R(0)},
$$

where $R(B)$ and $R(0)$ are the resistivities in a magnetic field with induction $B$ and without magnetic field, respectively. The error of $M R$ and $\sigma_{\square}(T)$ was within $5 \%$ and was mainly induced by the dimension measurement errors for the specimen and the electric contacts.

\section{Results and discussion}

Typical SEM images of the graphene specimens (Fig. 2a) with deposited Co particles $\left(\mathrm{Co}-\mathrm{Gr} / \mathrm{SiO}_{2}\right)$ show that the average size $\langle d\rangle$ of the particles was close to $200 \mathrm{~nm}$. $\mathrm{X}$-ray photoelectron spectroscopy (XPS) of the $\mathrm{Co}-\mathrm{Gr} /$ $\mathrm{SiO}_{2}$ specimen shows that the metallic particles form $\mathrm{Co}$ core / CoO shell structures $[33,34]$.
The structures of the source twisted graphene and the $\mathrm{Co}-\mathrm{Gr} / \mathrm{SiO}_{2}$ specimen with deposited Co particles were studied using selected area Raman spectroscopy. Detailed analysis of the Raman spectra of the specimens was reported earlier [14, 33-36]. The spatial distribution of the $I_{\mathrm{G}} / I_{\mathrm{D}}$ peak intensity ratio for the $\mathrm{Co}-\mathrm{Gr} / \mathrm{SiO}_{2}$ specimen is shown in Fig. $2 b$. The $I_{\mathrm{G}} / I_{\mathrm{D}}$ peak intensity ratio histograms shown in Fig. $2 b$ and $c$ indicate a higher defect density of twisted graphene after cobalt deposition as compared to the as-synthesized $\mathrm{G} / \mathrm{SiO}_{2}$ specimen.

Figure 3 shows the layer conductivity as a function of temperature $\sigma(T)$ for the $G / \mathrm{SiO}_{2}$ specimen (Curve 1 ) and the $\mathrm{Co}-\mathrm{G} / \mathrm{SiO}_{2}$ specimen (Curve 2) in a zero magnetic field. The pattern of Curves 1 and 2 showing an increase in the conductivity with temperature suggests an activation electrotransport mechanism. Furthermore the $\sigma_{\square}(T)$ functions become linear in semilogarithmic coordinates at below $150-200 \mathrm{~K}$.

The presence of the linear portions in the $\sigma_{\square}(\log [T])$ coordinates is usually attributed to an interference contribution to Drude conductivity under weak localization conditions [12, 15-18, 27, 37-40].

Figure 3 indicates a transition to conductivity saturation at below 5-6 K. This can be accounted for by the fact that the carrier free path in polycrystalline CVD graphene tends to grain size with a decrease in temperature [41]. As a result the conductivity may become temperature-independent due to carrier scattering at grain boundaries. Furthermore as shown earlier [14] the high defect density of twisted graphene may necessitate taking into account the contribution of Mott's 2D hopping mechanism at above $10 \mathrm{~K}$.

Raman scattering and SEM data suggest that Co particle deposition onto a twisted graphene layer increases the defect density of the $\mathrm{Co}-\mathrm{G} / \mathrm{SiO}_{2}$ specimen in comparison with source $G / \mathrm{SiO}_{2}$. Nevertheless comparison of Curves 1
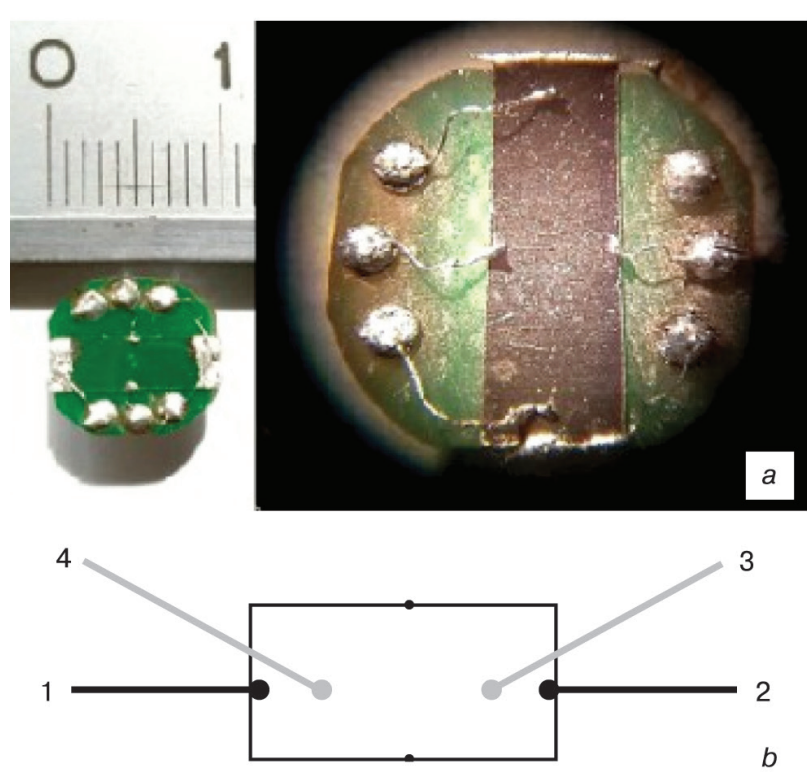

Figure 1. (a) Photo of specimen on contact pad and $(b)$ schematic of measurement probe arrangement: ( 1 and 2$)$ current contacts and (3 and 4$)$ voltage contacts. 

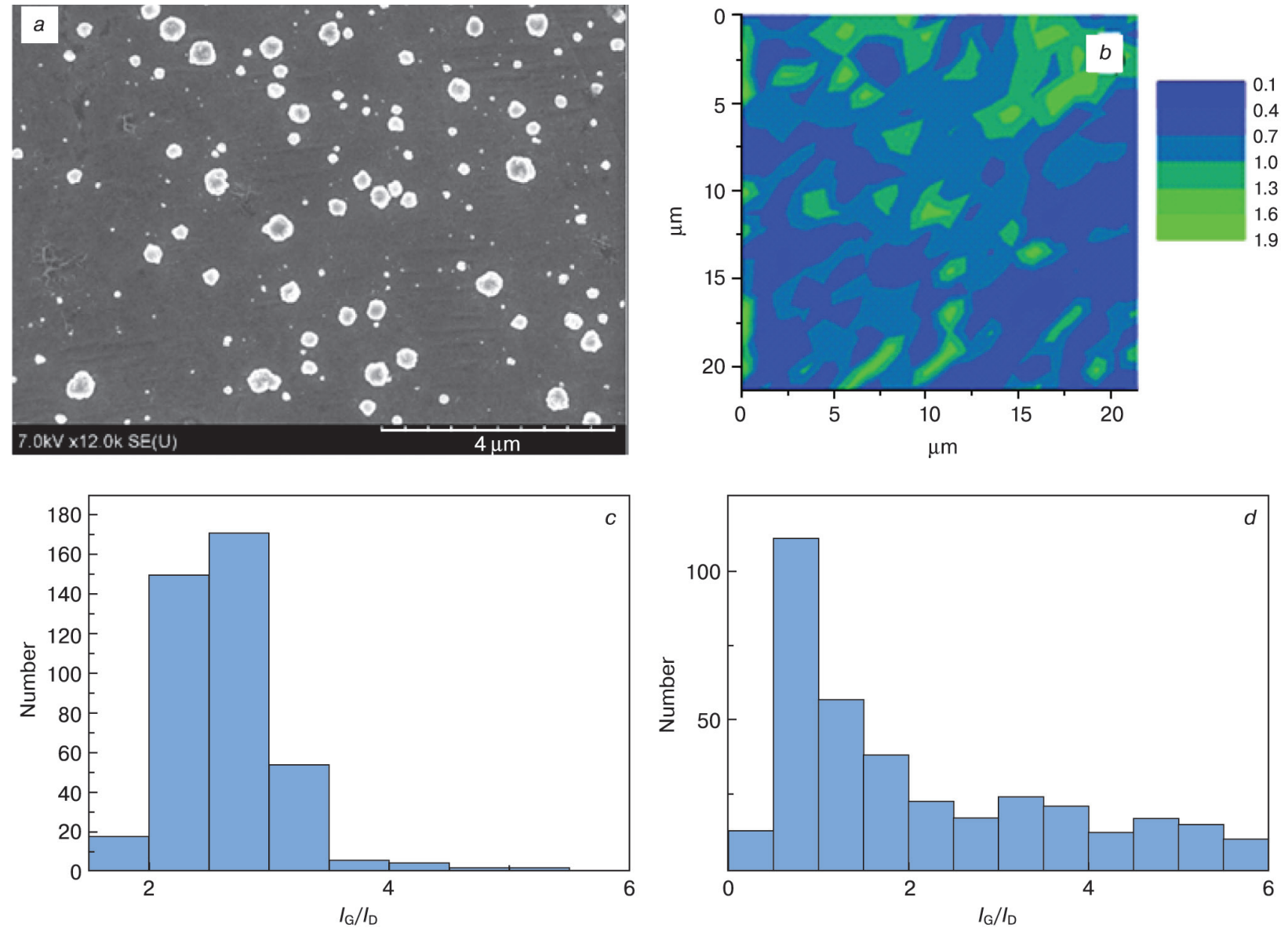

Figure 2. (a) Example of SEM image and (b) map of band intensity ratio $I_{\mathrm{G}} / I_{\mathrm{D}}$ for $\mathrm{Co}-G / \mathrm{SiO}$ specimen, and histograms of Raman spectra band intensity ratio $I_{\mathrm{G}} / I_{\mathrm{D}}$ for $(c) G / \mathrm{SiO}_{2}$ and $(d) \mathrm{Co}-G / \mathrm{SiO}_{2}$.

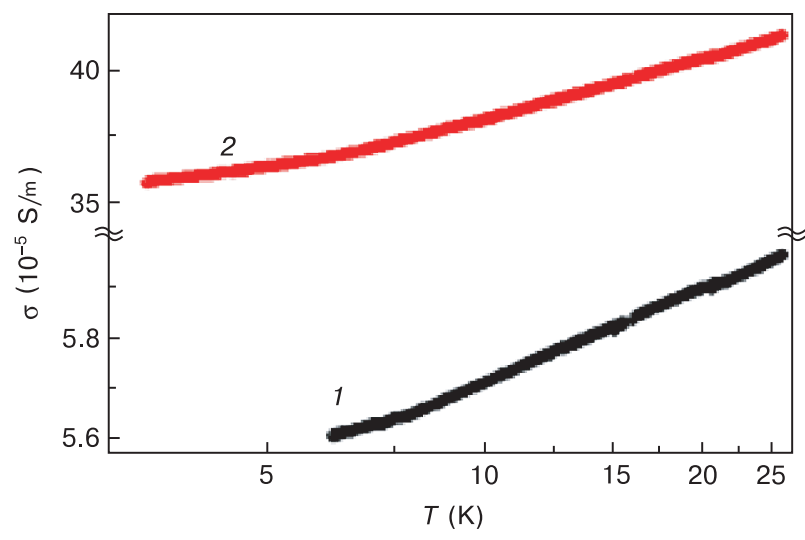

Figure 3. Layer conductivity as a function of temperature $\sigma_{(}(T)$ in semilogarithmic coordinates for (1) $\mathrm{G} / \mathrm{SiO}_{2}$ and (2) $\mathrm{Co}-\mathrm{G} /$ $\mathrm{SiO}_{2}$.

and 2 in Fig. 3 clearly indicates a decrease in the resistance after Co particle deposition. This testifies to the formation of a good (barrier-free) electric contact between the Co particles and the graphene layer. Thus as noted earlier $[14,31]$ metallic clusters bridge the underlying grain boundaries in the initial twisted polycrystalline graphene layer. Thus the XPS-detected $\mathrm{CoO}$ shell is only present on the top of the cobalt particles and does not isolate the cobalt/graphene interface.

One can reasonably assume that cobalt particle deposition can similarly affect the carrier phase break length at the crossing with the graphene/Co particle interfaces. The phase break time determined for a $\mathrm{Co}-G / \mathrm{SiO}$, specimen from the $\sigma_{(}(T)$ temperature functions [14] was one order of magnitude greater than that for the source $G / \mathrm{SiO}_{2}$ specimen. However the cited work only dealt with the interference contribution to Drude conductivity for weak localization [38, 40].

To reveal other potential contributions to magnetotransport we analyzed the relative magnetoresistance as a function of magnetic field $M R(B, T)$ (Fig. 4). The following three important features of these $M R(B, T)$ functions are worth special mentioning. First, both specimens exhibit a competition between the negative and positive magnetoresistance effect contributions at low temperatures. Secondly, the negative magnetoresistance contribution at below $50 \mathrm{~L}$ only dominates in magnetic fields of below $1 \mathrm{Tl}$. Furthermore Co particle deposition reduces the negative magnetoresistance contribution as follows from Curves $1-4$ in Fig. 4. Thirdly, the positive magnetoresistance contribution to $M R(B, T)$ increases with an increase in both the magnetic induction $B$ and temperature. 

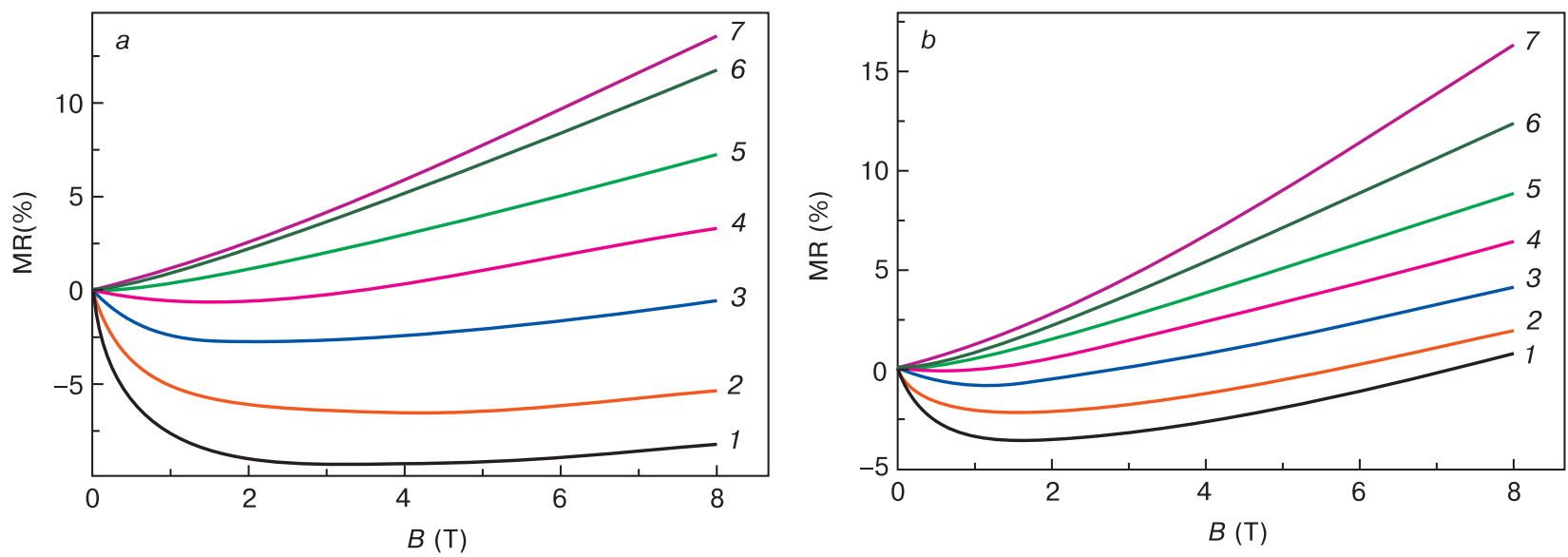

Figure 4. Relative magnetoresistance $M R$ for $(a) G / \mathrm{SiO}_{2}$ and $(b)$ Co- $G / \mathrm{SiO}_{2}$ specimens as a function of magnetic field induction $B$ at $T=(1) 5 \mathrm{~K}$, (2) $10 \mathrm{~K}$, (3) $25 \mathrm{~K}$, (4) $50 \mathrm{~K}$, (5) $100 \mathrm{~K}$, (6) $200 \mathrm{~K}$ and (7) $300 \mathrm{~K}$.

As a result the negative magnetoresistance contribution is completely suppressed at $T>100-120 \mathrm{~K}$ and only positive magnetoresistance effect is observed. $M R(B)$ of the $\mathrm{Co}-\mathrm{G} / \mathrm{SiO}_{2}$ specimens (Curves $5-7$ in Fig. $3 a$ ) increases with temperature and magnetic field much more rapidly than for the source $G / \mathrm{SiO}_{2}$ specimen.

These regularities of $M R(B, T)$ behavior at $2-300 \mathrm{~K}$ suggest that the test graphene specimens may exhibit not only weak localization corrections (providing negative magnetoresistance effect in fields of less than $1 \mathrm{Tl}$ $[38,40]$ ) but also other contributions to quantum interference conductivity corrections which produce positive magnetoresistance effect. There are indications [18] that positive magnetoresistance effect in graphene entails domination of intervalley scattering, pseudospin chirality violation and graphene buckling corrections [38-40]. These contributions to graphene conductivity as a function of magnetic field $\Delta \sigma(B)=[\sigma(0)-\sigma(B)]$, along with the localization correction, are usually described with relationships like

$\Delta \sigma_{\square}=\frac{e^{2}}{\pi h}\left[F\left(\frac{B}{B_{\varphi}}\right)-F\left(\frac{B}{B_{\varphi}+2 B_{i}}\right)-2 F\left(\frac{B}{B_{\varphi}+B_{\square}}\right)\right]$,

where

$$
F(x)=\ln (x)+\psi\left(0,5+x^{-1}\right),
$$

$\psi$ is the digamma function. Here the $x=B / B_{\varphi, i *}$ parameter is determined by the ratio between the induction of the external magnetic field $B$ and that of the characteristic field $B_{\varphi, i, *}$ of scattering. The characteristic fields $B_{\varphi, i, *}$ determine the carrier phase break times $\tau_{\varphi, i, *}$ for the respective process of elastic or quasi-elastic scattering. These phase break times are determined from the relationship

$$
\tau_{\varphi, i, *}=\frac{\hbar c}{4 e D} B_{\varphi, i, *}^{-1}
$$

where $D$ is the electron diffusion coefficient.

The first term in Eq. (3) with the index $\varphi$ corresponds to scattering at low-energy phonons, the second term with the index $i$ corresponds to intervalley scattering and the third term with the index * corresponds to chirality violation and graphene buckling induced by thermal fluctuations.

To evaluate the characteristic times of the processes leading to the formation of quantum corrections to Drude conductivity (Eq. (4)) one should calculate the carrier diffusion coefficient $D$. Earlier, Tikhonenko [38] proposed the following expression for diffusion coefficient $D$ in graphene:

$$
D=v_{F} \frac{l}{2}
$$

where $v_{F}$ is the Fermi carrier velocity, $l$ is the free path equal to $l=h /\left(2 e^{2} k_{F} n\right), k_{F}$ is the Fermi pulse and $n$ is the carrier concentration. It is assumed that only the carriers at the Fermi surface participate in conductivity. Thus this approach is the most correct for defect-free graphene at low temperatures.

Since quantum corrections exist in the test graphene layers at far above $25 \mathrm{~K}$ [26] we suggest a further generalized $D$ calculation approach which can be also used for high temperatures.

We represent a conducting specimen as a homogeneous medium and ignore the contribution of large defects, e.g. grain boundaries. We further assume that the carrier energy distribution is described by the Fermi-Dirac function

$$
f(E)=\frac{1}{e^{\frac{E-\mu}{k T}}+1} .
$$

Then the carrier concentration can be described as follows:

$$
n=\int f(E) g(E) \mathrm{d} E
$$

where $g(E)$ is the band density of states. We use the expression 


$$
\sigma=e^{2} D\left(\frac{\partial n}{\partial \mu}\right)_{T},
$$

borrowed from earlier work [43] to interrelate the conductivity and the diffusion coefficient. Here $(\partial n / \partial \mu)_{T}$ describes the carriers participating in charge transport. Since the chemical potential $\mu$ is not component of the density of states $g(E)$, Eq. (7) can be transformed to

$$
\begin{aligned}
& \sigma=e^{2} D \int_{-\infty}^{+\infty} g(E) \frac{\partial}{\partial \mu}\left(\frac{1}{e^{\frac{E-\mu}{k T}}+1}\right) \mathrm{d} E= \\
& =\frac{e^{2} D}{k T} \int_{0}^{\infty} g(E)\left(\frac{e^{\frac{E-\mu}{k T}}}{\left(e^{\frac{E-\mu}{k T}}+1\right)^{2}}\right) \mathrm{d} E= \\
& =\frac{e^{2} D}{k T} \int_{0}^{\infty} g(E) f(E)(1-f(E)) \mathrm{d} E .
\end{aligned}
$$

This latter expression yields the sought formula of the diffusion coefficient:

$$
D=\frac{\sigma k T}{e^{2}} \int_{0}^{\infty} f(E, \mu)[1-f(E, \mu)] g(E) \mathrm{d} E .
$$

Equation (9) allows comparing the average (effective) carrier diffusion coefficient.

The diffusion coefficient calculation method proposed herein gives the following important advantages: (a) possibility of evaluating $D$ by changing the density of states $g(E)$ for either single-layer or two-layered graphene; (b) possibility of taking into account carrier energy distributions at temperatures far above LH; (c) possibility of taking into account Fermi level shift (chemical potential) $\mu$ upon application of an external electric field to specimen (e.g. for measurements with a third electrode).

The main limitation upon the use of this method is the fact that Eq. (8) which correlates $\sigma$ and $D$ is only valid if diffusion (drift) conductivity is dealt with. At low temperatures the experimentally measured conductivity $\sigma$ can incorporate a hopping transport mechanism contribution. Therefore when evaluating the diffusion coefficient $D$ one should make sure that there is no large hopping conductivity contribution in the specimen or it is possible to distinguish the hopping and drift (diffusion) conductivity contributions.

From the negative magnetoresistance effect as a function of magnetic field in accordance with Eqs. (3) and (4), one can determine the phase break time due to weak localization as a function of temperature $\tau_{\phi}(T)[15-18,39]$. The parameter $D$ in Eq. (4) was evaluated using Eq. (8) to be $0.018 \mathrm{~m}^{2} / \mathrm{s}$ for the $G / \mathrm{SiO}_{2}$ specimen and $0.025 \mathrm{~m}^{2} / \mathrm{s}$ for $\mathrm{Co}-\mathrm{G} / \mathrm{SiO}_{2}$. The dependences shown in Fig. 5 suggest that the pattern of the $\tau_{0}(T)$ curves at below $10 \mathrm{~K}$ can be

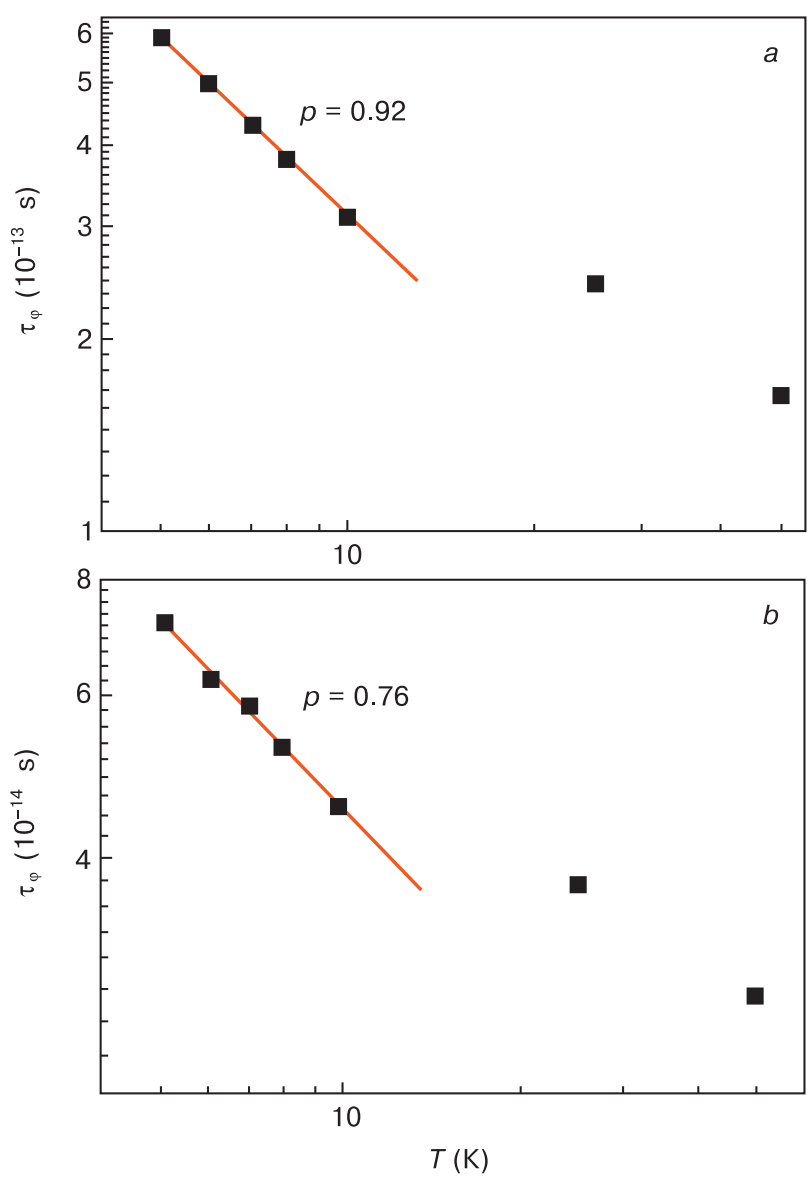

Figure 5. Phase break time $\tau_{\varphi}(T)$ as a function of temperature for quantum correction contribution due to weak localization in (a) $\mathrm{G} / \mathrm{SiO}_{2}$ and (b) $\mathrm{Co}-\mathrm{G} / \mathrm{SiO}_{2}$ specimens. Dots: calculated from experimental $\Delta \sigma(B)$ functions; lines: linear approximation.

described in accordance with the quantum correction theory as a power function like $\tau_{\varphi}(T) \sim T^{p p}$ with the exponents $p \approx 0,92$ for the $G / \mathrm{SiO}_{2}$ specimen and $p \approx 0.76$ for the $\mathrm{Co}-\mathrm{G} / \mathrm{SiO}_{2}$ specimen. The small difference between the exponent for the source $\mathrm{G} / \mathrm{SiO}_{2}$ specimen and the theoretical value $p=1$ confirms that the phase break at negative magnetoresistance effect originates mainly from the elastic electron scattering at low-energy phonons [37, 39]. The decrease of the exponent $p$ and the phase break time $\tau_{\varphi}$ as a result of cobalt particle deposition seems to result from grain boundary bridging by the cobalt particles.

Unfortunately, deriving characteristic process times for other scattering types which give positive contributions to quantum corrections at positive magnetoresistance effect $[39,40]$ is a difficult task. One reason is that the characteristic magnetic fields that are terms of this relationship incorporate the contributions $\left(B_{\varphi}+2 B_{i}\right)$ and $\left(B_{\varphi}+B_{*}\right)$ from two processes, and the inverse scattering time $\tau_{*}^{-1}$, which accounts for thermal fluctuations in graphene and is incorporated in the characteristic field $B_{*}$, is the sum of the inverse scattering times relating to chirality violation and intervalley scattering $\left(\tau_{*}^{-1}=\tau_{w}^{-1}+\tau_{i}^{-1}\right)$. Therefore Fig. 6 shows the ratios of the phase break time $\tau_{\varphi}$ (as determined above) to the intervalley scattering characteristic time $\tau_{I}$ and the chirality violation and buckling characteristic 


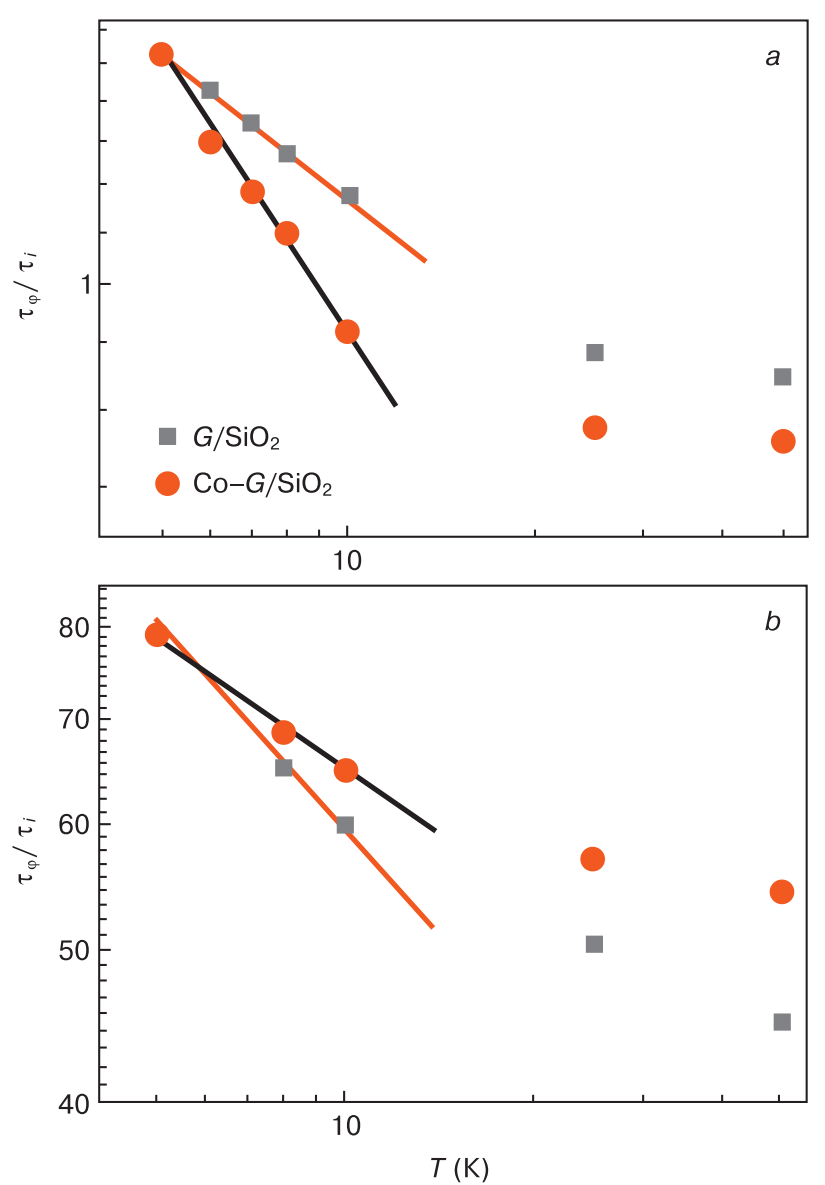

Figure 6. Temperature dependences of ratios between phase break time $\tau_{\varphi}$ due to low-energy phonon scattering and phase break times for $(a)$ intervalley scattering $\tau_{i}$ and $(b)$ cxhirality violation $\tau_{*}$ in $G / \mathrm{SiO}_{2}$ and $\mathrm{Co}-G / \mathrm{SiO}_{2}$. Dots: calculated from experimental $\Delta \sigma(B)$ functions; lines: linear approximation.

time $\tau_{*}$, as functions of temperature. It can be seen that the characteristic phase break times for intervalley scattering $\tau_{i}$ in graphene without Co are approximately two times shorter than the phase break time $\tau_{\varphi}$ for weak localization. In the meantime the characteristics phase break times due to chirality violation $\tau_{*}$ decrease by almost two orders of magnitude as compared to $\tau_{\varphi}$. Furthermore cobalt particle deposition reduces both $\tau_{i}$, and $\tau_{*}$ in comparison with those of source graphene.

Noteworthy, the temperature dependence of these ratios also has the form of a power function $\tau_{i}, \tau_{*} \sim T^{-p}$ in the $2-10 \mathrm{~K}$ range as indicated by the linear portions of the curves in double logarithmic coordinates. After cobalt deposition the line slope increases for $\tau_{*}$ (from $p \approx 0.38$ to $p \approx 0.77$ ) and decreases for $\tau_{i}$ (from $p \approx 0.42$ to $p \approx 0.29$ ). This behavior agrees with earlier results for single-layer graphene [42].

\section{Conclusion}

We showed that the $G / \mathrm{SiO}_{2}$ and $\mathrm{Co}-G / \mathrm{SiO}_{2}$ structures exhibit a competition between negative and positive magnetoresistance effects at low temperatures. Negative magnetoresistance effect is completely suppressed by weak magnetic fields $(B \leq 1 \mathrm{Tl})$, and Co particle deposition onto the graphene layer reduces the contribution of negative magnetoresistance effect. Low-temperature electrotransport and magnetotransport at negative magnetoresistance effect are mainly caused by the localization quantum correction to Drude conductivity. In magnetic fields below $1 \mathrm{Tl}$ (positive magnetoresistance effect) the dominating quantum correction contributions are those from intervalley scattering, chirality violation and graphene buckling. Cobalt particle deposition slightly increases the phase break time $\tau$ due to weak localization whereas the characteristic phase break times due to intervalley scattering $\tau_{i}$ and chirality violation $\tau_{*}$, on the contrary, decrease strongly.

\section{Acknowledgements}

The work was accomplished with financial support from the State Program for Photonics, Opto- and Microelectronics (Assignment No. 3.3.01), the State Committee for Science and Technology of the Republic of Belarus (BRFFI Project No. F18PLSHG-005) and the Joint Nuclear Research Institute (Russian Federation) Contract No. 08626319/182161170-74. S.L. Prischepa and I.V. Komissarov are grateful to the National Nuclear Research University MIFI for financial support of the Competitiveness Upgrading Program. The Authors express gratitude to Ph.D. (Phys.) I.A. Svito, Belarus State University, for measuring electric properties and Postgraduate Student A.V. Pashkevich, Institute for Nuclear Physics, Belarus State University, for help with article preparation.

\section{References}

1. Ferrari A.C., Bonaccorso F., Fal'ko V., Novoselov K.S., Roche S., Bøggild P. et al. Science and technology roadmap for graphene, related two-dimensional crystals, and hybrid systems. Nanoscale, 2015; 7(11): 4598-4810. https://doi.org/10.1039/C4NR01600A

2. Liu Y., Liu Z., Lew W.S., Wang Q.J. Temperature dependence of the electrical transport properties in few-layer graphene interconnects. Nanoscale Res. Lett., 2013; 8: 335-340. https://doi.org/10.1186/1556276X-8-335
3. Castro Neto A.H., Guinea F., Peres N.M.R., Novoselov K.S., Geim A.K. The electronic properties of grapheme. Rev. Mod. Phys., 2009; 81(1): 109-115. https://doi.org/10.1103/RevModPhys.81.109

4. Asshoff P.U., Sambricio J.L., Rooney A.P., Slizovskiy S., Mishchenko A., Rakowski A.M., Hill E.W., Geim A.K., Haigh S.J., Fal'ko V.I., Vera-Marun I.J., Grigorieva I.V. Magnetoresistance of vertical Co-graphene-NiFe junctions controlled by charge transfer and 
proximity-induced spin splitting in graphene. 2 D Mater., 2017; 4(3): 031004. https://doi.org/10.1088/2053-1583/aa7452

5. Iqbal M.Z., Iqbal M.W., Lee J.H., Kim Y.S., Chun S.-H., Eom J. Spin valve effect of $\mathrm{NiFe} / \mathrm{graphene} / \mathrm{NiFe}$ junctions. Nano Research., 2013; 6: 373-380. https://doi.org/10.1007/s12274-013-0314-x

6. De Franco V.C., Castro G.M.B., Corredor J., Mendes D., Schmidt J. E. In-situ magnetization measurements and ex-situ morphological analysis of electrodeposited cobalt onto chemical vapor deposition graphene/ $\mathrm{SiO}_{2} / \mathrm{Si}$. Carbon Lett., 2017; 21: 16-22. https://doi. org/10.5714/CL.2017.21.016

7. Khatami Y., Li H., Xu C., Banerjee K. Metal-to-multilayer-graphene contact. Part I: Contact resistance modeling. IEEE Trans. Electron. Devices, 2012; 59(9): 2444-2452. https://doi.org/10.1109/ TED.2012.2205256

8. Ruhl G., Wittmann S., Koenig M., Neumaier D. The integration of graphene into microelectronic devices. Beilstein J. Nanotechnol., 2017; 8: 1056-1064. https://doi.org/10.3762/bjnano.8.107

9. Bayev V.G., Fedotova J.A., Kasiuk J.V., Vorobyova S.A., Sohor A.A., Komissarov I.V., Kovalchuk N.G., Prischepa S.L., Kargin N.I., Andrulevičius M., Przewoznik J., Kapusta Cz., Ivashkevich O.A., Tyutyunnikov S.I., Kolobylina N.N., Guryeva P.V. CVD graphene sheets electrochemically decorated with «core-shell» $\mathrm{Co} /$ CoO nanoparticles. Appl. Surf. Sci., 2018; 440: 1252-1260. https:// doi.org/10.1016/j.apsusc.2018.01.245

10. Tuček J., Sofer Z., Bouša D., Pumera M., Holá K., Malá A., Poláková K., Havrdová M., Čépe K., Tomanec O., Zbořil R. Air-stable superparamagnetic metal nanoparticles entrapped in graphene oxide matrix. Nature Commun., 2016; 7: 12879. https://doi.org/10.1038/ ncomms 12879

11. Zhidkov I.S., Skorikov N.A., Korolev A.V., Kukharenko A.I., Kurmaev E.Z., Fedorov V.E., Cholakh S.O. Electronic structure and magnetic properties of graphene/Co composite. Carbon, 2015; 91: 298-303. https://doi.org/10.1016/j.carbon.2015.04.086

12. Sokolik A.A., Zabolotskiy A.D., Lozovik Yu. E. Many-body effects of Coulomb interaction on Landau levels in graphene. Phys. Rev. B, 2017; 95(12): 125402-1-4. https://doi.org/10.1103/PhysRevB.95.125402

13. Majumder C., Bhattacharya S., Saha S.K. Anomalous large negative magnetoresistance in transition-metal decorated graphene: Evidence for electron-hole puddles. Phys. Rev. B, 2019; 99(4): 045408-1-13. https://doi.org/10.1103/PhysRevB.99.045408

14. Fedotov A.K., Prischepa S.L., Fedotova J.A., Bayev V.G., Ronassi A.A., Komissarov I.V., Kovalchuk N.G., Vorobyova S.A., Ivashkevich O.A. Electrical conductivity and magnetoresistance in twisted graphene electrochemically decorated with Co particles. Physica E: Low-dimensional Systems and Nanostructures, 2020; 117: 113790 https://doi.org/10.1016/j.physe.2019.113790

15. Jobst J., Waldmann D., Gornyi I.V., Mirlin A.D., Weber H.B. Electron-electron interaction in the magnetoresistance of graphene. Phys. Rev. Lett., 2012; 108(10): 106601. https://doi.org/10.1103/PhysRevLett.108.106601

16. Morozov S.V., Novoselov K.S., Katsnelson M.I., Schedin F., Ponomarenko L.A., Jiang D., Geim A.K. Strong suppression of weak localization in graphene. Phys. Rev. Lett., 2006; 97(1): 016801-1-4. https://doi.org/10.1103/PhysRevLett.97.016801

17. Gorbachev R.V., Tikhonenko F.V., Mayorov A.S., Horsell D.W., Savchenko A.K. Weak localization in bilayer grapheme. Phys. Rev. Lett., 2007; 98(17): 176805-1-4. https://doi.org/10.1103/PhysRevLett.98.176805
18. Kechedzhi K., McCann E., Fal'ko V.I., Suzuura H., Ando T., Altshuler B.L. Weak localization in monolayer and bilayer grapheme. Eur. Phys. J. Spec., 2007; 148: 39-54. https://doi.org/10.1140/epjst/e2007-00224-6 19. Shlimak I., Butenko A.V., Zion E., Richter V., Kaganovski Yu., Wolfson L., Sharoni A., Haran A., Naveh D., Kogan E., Kaveh D. Structure and electron transport in irradiated monolayer graphene. In: Future Trends in Electronics: Journey into Unknown. John Wiley \& Sons, Inc.: Hoboken (New Jersey), 2016: 217-231. https://doi. org/10.1002/9781119069225.ch2-9

20. Shlimak I., Haran A., Zion E., Havdala T., Kaganovskii Yu., Butenko A.V., Wolfson L., Richter V., Naveh D., Sharoni A., Kogan E., Kaveh M. Raman scattering and electrical resistance of highly disordered graphene. Phys. Rev., 2015; 91(4): 045414-1-4. https://doi. org/10.1103/PhysRevB.91.045414

21. Shlimak I., Zion E., Butenko A.V., Wolfson L., Richter V., Kaganovskii Yu., Sharoni A., Haran A., Naveh D., Kogan E., Kaveh M. Hopping magnetoresistance in ion irradiated monolayer graphene. Physica E: Low-dimensional Systems and Nanostructures, 2016; 76: 158-163. https://doi.org/10.1016/j.physe.2015.10.025

22. Isacsson A., Cummings A. W., Colombo L., Colombo L., Kinaret J.M., Roche S. Scaling properties of polycrystalline graphene: a review. 2D Mater., 2017; 4(1): 012002-1-13. https://doi. org/10.1088/2053-1583/aa5147

23. Huang P.Y., Ruiz-Vargas C.S., van der Zande A.M., Whitney W.S., Levendorf M.P., Kevek J.W., Garg S., Alden J.S., Hustedt C.J., Zhu Y., Park J., McEuen P.L., Muller D.A. Grains and grain boundaries in single-layer graphene atomic patchwork quilts. Nature, 2011; 469: 389-392. https://doi.org/10.1038/nature09718

24. Wang C., Wang J., Barber A.H. Stress concentrations in nanoscale defective grapheme. AIP Advance, 2017; 7(11): 115001. https://doi. org/10.1063/1.4996387

25. Lebedev A.A., Agrinskaya N.V., Lebedev S.P., Mynbaeva M.G., Petrov V.N., Smirnov A.N., Strel'chuk A.M., Titkov A.N., Shamshur D.V. Low-temperature transport properties of multigraphene films grown on the $\mathrm{SiC}$ surface by sublimation. Semiconductors, 2011; 45: 623-627. https://doi.org/10.1134/S1063782611050186

26. Ramnani P., Neupane M.R., Ge S., Balandin A.A., Lake R.K., Mulchandani A. Raman spectra of twisted CVD bilayer grapheme. Carbon, 2017; 123: 302-306. https://doi.org/10.1016/j.carbon.2017.07.064

27. Altshuler B.L., Aronov A.G., Khmelnitsky D.E. Effects of electron-electron collisions with small energy transfers on quantum localisation. J. Phys. C: Solid State Phys., 1982; 15(36): 7367-7386. https://doi.org/10.1088/0022-3719/15/36/018

28. Shklovskii B.I., Efros A.L. Electronic properties of doped semiconductors. Springer Series in Solid-State Sciences. Vol. 45. Berlin; Heidelberg: Springer-Verlag, 1984, 388 p. https://doi.org/10.1007/9783-662-02403-4

29. Shklovskii B.I. Hopping conductivity of semiconductors in strong magnetic fields. JETP, 1972; 34(5): 1084-1088. URL: http://www. jetp.ac.ru/cgi-bin/dn/e_034_05_1084.pdf

30. Mikoshiba N. Weak-field magnetoresistance of hopping conduction in simple semiconductors. J. Phys. Chem. Solids, 1963; 24(3): 341346. https://doi.org/10.1016/0022-3697(63)90192-6

31. Bayev V., Fedotova J., Humennik U., Vorobyova S., Konakow A., Fedotov A., Svito I., Rybin M., Obraztsova E. Modification of electric transport properties of CVD graphene by electrochemical deposition of cobalt nanoparticles. Intern. J. Nanoscience, 2019; 18(03n04): 1940041-1-4. https://doi.org/10.1142/S0219581X19400416 
32. Solin S.A., Tineke Thio, Hines D.R., Heremans J.J. Enhanced room-temperature geometric magnetoresistance in inhomogeneous narrow-gap semiconductors. Science, 2000; 289(5484): 1530-1532. https://doi.org/10.1126/science.289.5484.1530

33. Komissarov I.V., Kovalchuk N. G., Labunov V. A., Girel K. V., Korolik O. V., Tivanov M. S., Lazauskas A., Andrulevičius M., Tamulevičius T., Grigaliūnas V., Meškinis Š., Tamulevičius S., Prischepa S. L. Nitrogen-doped twisted graphene grown on copper by atmospheric pressure CVD from a decane precursor. Beilstein J. Nanotechnol., 2017; 8: 145-158. https://doi.org/10.3762/bjnano.8.15

34. Kovalchuk N.G., Nigerish K.A., Mikhalik M.M., Kargin N.I., Komissarov I.V., Prischepa S.L. Possibility of determining the graphene doping level using Raman spectra. J. Appl. Spectrosc. 2018; 84: 995-998. https://doi.org/10.1007/s10812-018-0576-x

35. Chung T.-F., Xu Y., Chen Y.P. Transport measurements in twisted bilayer graphene: Electron-phonon coupling and Landau level crossing. Phys. Rev. B, 2018; 98(3): 035425. https://doi.org/10.1103/ PhysRevB.98.035425

36. Shih C.-J., Vijayaraghavan A., Krishnan R., Sharma R., Han J.-H., Ham M.-H., Jin Z., Lin S., Paulus G.L.C., Reuel N.F., Wang Q.H., Blankschtein D., Strano M.S. Bi- and trilayer graphene solutions. Nat. Nanotechnol., 2011; 6(7): 439-445. https://doi.org/10.1038/ nnano.2011.94

37. Pudalov V.M. Metallic conduction, apparent metal-insulator transition and related phenomena in two-dimensional electron liquid.
In: Proceedings of the International School of Physics «Enrico Fermi». Vol. 157: The Electron Liquid Paradigm in Condensed Matter Physics. IOS Press, 2004: 335-356. https://doi.org/10.3254/978-161499-013-0-335

38. Tikhonenko F.V., Horsell D.W., Gorbachev R.V., Savchenko A.K. Weak localization in graphene flakes. Phys. Rev. Lett., 2008; 100(5): 056802. https://doi.org/10.1103/PhysRevLett.100.056802

39. McCann E., Kechedzhi K., Fal'ko V.I., Suzuura H., Ando T., Altshuler B.L. Weak-localization magnetoresistance and valley symmetry in graphene. Phys. Rev. Lett., 2006; 97(14): 146805. https://doi. org/10.1103/PhysRevLett.97.146805

40. Kechedzhi K., Fal'ko V.I., McCann E., Altshuler B.L. Influence of trigonal warping on interference effects in bilayer graphene. Phys. Rev. Lett., 2007; 98(17): 176806. https://doi.org/10.1103/PhysRevLett.98.176806

41. Tikhonenko F.V., Kozikov A.A., Savchenko A.K., Gorbache R.V. Transition between electron localization and antilocalization in graphene. Phys. Rev. Lett., 2009; 103(22): 226801-1-4. https://doi. org/10.1103/PhysRevLett.103.226801

42. Araujo E.N.D., Brant J.C., Archanjo B.S., Medeiros-Ribeiro G., Alves E.S. Quantum corrections to conductivity in graphene with vacancies. Physica E: Low-dimensional Systems and Nanostructures, 2018; 100: 40-44. https://doi.org/10.1016/j.physe.2018.02.025

43. Bonch-Bruevich V.L., Kalashnikov S.G. Semiconductor physics. Moscow: Nauka, 1977. 674 p. (In Russ.) 